\title{
Preconditioning on subspace quasi-Newton method for large scale unconstrained optimization
}

\begin{abstract}
Recently, subspace quasi-Newton (SQN) method has been widely used in solving large scale unconstrained optimization. Besides constructing sub-problems in low dimensions so that the storage requirement as well as computational cost can be reduced, it can also be implemented extremely fast when the objective function is a combination of computationally cheap nonlinear functions. However, the main deficiency of SQN method is that it can be very slow on certain type of non-linear problem. Hence, a preconditioner which is computationally cheap and is a good approximation to the actual Hessian is constructed to speed up the convergence of the quasi-Newton methods since the evaluation of the actual Hessian is considered as impractical and costly. For this purpose, a diagonal updating matrix has been derived to replace the identity matrix in approximating the initial inverse Hessian. The numerical results show that the preconditioned SQN method performs better than the standard SQN method that without preconditioning.
\end{abstract}

Keyword: Preconditioned; Subspace method; Quasi-Newton method; Large scale; Unconstrained optimization 